\title{
Single-cell transcriptome sequencing on the Nanopore platform with ScNapBar
}

\author{
QI WANG, ${ }^{1}$ SVEN BÖNIGK, ${ }^{1}$ VOLKER BÖHM, ${ }^{2,3}$ NIELS GEHRING, ${ }^{2,3}$ JANINE ALTMÜLLER, ${ }^{4}$ \\ and CHRISTOPH DIETERICH ${ }^{1,5,6}$ \\ ${ }^{1}$ Klaus Tschira Institute for Integrative Computational Cardiology, University Hospital Heidelberg, 69120 Heidelberg, Germany \\ ${ }^{2}$ Institute for Genetics, University of Cologne, 50674 Köln, Germany \\ ${ }^{3}$ Center for Molecular Medicine Cologne (CMMC), University of Cologne, 50937 Köln, Germany \\ ${ }^{4}$ Cologne Center for Genomics (CCG), University of Cologne, 50931 Köln, Germany \\ ${ }^{5}$ Department of Internal Medicine III (Cardiology, Angiology, and Pneumology), University Hospital Heidelberg, 69120 Heidelberg, Germany \\ ${ }^{6}$ German Centre for Cardiovascular Research (DZHK)-Partner Site Heidelberg/Mannheim, 69120 Heidelberg, Germany
}

\begin{abstract}
The current ecosystem of single-cell RNA-seq platforms is rapidly expanding, but robust solutions for single-cell and singlemolecule full-length RNA sequencing are virtually absent. A high-throughput solution that covers all aspects is necessary to study the complex life of mRNA on the single-cell level. The Nanopore platform offers long read sequencing and can be integrated with the popular single-cell sequencing method on the 10x Chromium platform. However, the high error-rate of Nanopore reads poses a challenge in downstream processing (e.g., for cell barcode assignment). We propose a solution to this particular problem by using a hybrid sequencing approach on Nanopore and Illumina platforms. Our software ScNapBar enables cell barcode assignment with high accuracy, especially if sequencing saturation is low. ScNapBar uses unique molecular identifier (UMI) or Naïve Bayes probabilistic approaches in the barcode assignment, depending on the available Illumina sequencing depth. We have benchmarked the two approaches on simulated and real Nanopore data sets. We further applied ScNapBar to pools of cells with an active or a silenced nonsense-mediated RNA decay pathway. Our Nanopore read assignment distinguishes the respective cell populations and reveals characteristic nonsense-mediated mRNA decay events depending on cell status.
\end{abstract}

Keywords: Bayesian; 10x genomics; cell barcode assignment; nonsense-mediated mRNA decay (NMD)

\section{INTRODUCTION}

Full-length cDNA sequencing allows us to investigate the differential isoforms of transcripts, which is especially useful in studying the complex life of mRNA. Compared to the Illumina sequencing approaches, third-generation sequencing generates much longer reads and thus avoids artifacts from transcriptome assembly, but often has limitations such as low throughput and poor base-calling accuracy. Two principal third-generation sequencing platforms exist: Oxford Nanopore Technologies (ONT) and Pacific Biosciences (PacBio) (Volden et al. 2018). Others and we chose the ONT platform to study full-length mRNA transcripts due to its better scalability and flexibility (Lebrigand et al. 2020). Full-length transcriptome sequencing can be taken to the single level by sequencing barcoded 10× Ge-

Corresponding author: christoph.dieterich@uni-heidelberg.de Article is online at http://www.rnajournal.org/cgi/doi/10.1261/rna. 078154.120. Freely available online through the RNA Open Access option. nomics cDNA libraries. However, this brings about certain challenges, which we address in our work.

First, the native error rate of Nanopore DNA sequencing is $<5 \%$ on the latest R10.3 platform (http://nanoporetech .com) as opposed to the typical Illumina error rate of $0.1 \%$. Due to its high error rate, barcode identification and assignment are challenging for single-cell sequencing. In the 10x Genomics single-cell protocol, about $99 \%$ barcode sequences from Illumina sequencing can be exactly matched to the 16-bp cell barcodes, while with Nanopore sequencing, the exact matches are $<50 \%$ (0.99916 vs. 0.9516). Many experimental and computational approaches have been developed to correct Nanopore data. For example, the rolling circle to concatemeric consensus (R2C2) approach can produce two million fulllength cDNA sequences per MinION flow cell and

C 2021 Wang et al. This article, published in RNA, is available under a Creative Commons License (Attribution-NonCommercial 4.0 International), as described at http://creativecommons.org/licenses/ by-nc/4.0/. 
achieved $98 \%$ accuracy (Volden et al. 2018; Cole et al. 2020; Volden and Vollmers 2020). Single-cell Nanopore sequencing with UMls (ScNaUmi-seq) can assign cellular barcodes with 99.8\% accuracy (Lebrigand et al. 2020). However, R2C2 requires sufficient sequencing coverage to call consensus reads, and ScNaUmi-seq requires high sequencing depth to guarantee an adequate overlap of UMI sequences between Illumina and Nanopore libraries.

On the other hand, end-to-end solutions for barcode demultiplexing and read quality filtering on the ONT platform are still in its infancy. For example, Mandalorion uses BLAT (Kent 2002) for barcode demultiplexing (Byrne et al. 2017). Porechop (https:// github.com/rrwick/Porechop) uses SeqAn (Döring et al. 2008) for adapter removal and barcode demultiplexing in Nanopore sequencing, but it is based on the best alignment, which could be error-prone. Minibar (Krehenwinkel et al. 2019), Deep-binner (Wick et al. 2018), and DeePlexiCon (Smith et al. 2020) are only suitable for multiplexing a few barcoded samples rather than the single-cell library which contains several thousands of barcodes.

Therefore, we developed a software tool called ScNapBar (single-cell Nanopore barcode demultiplexer) that demultiplexes Nanopore barcodes and is particularly suited for low depth Illumina and Nanopore sequencing. We evaluated the performance of ScNapBar and demonstrated its high accuracy in cell barcode assignment for simulated and real Nanopore data. Our workflow is presented in Figure 1.

\section{RESULTS}

\section{Benchmarking the two ScNapBar run modes}

ScNapBar offers two run modes. The first one uses cell barcode and UMI information without any additional modeling aspect. The second one introduces a probabilistic model, which performs very well in cases of low sequencing saturation (i.e., UMI coverage in Illumina data).

\section{The UMI approach of ScNapBar}

The UMI approach requires a matching cell barcode and UMI tag and was first developed in Sicelore (Lebrigand et al. 2020). Any cell barcode predictions that are support- ed by the presence of both, barcode and UMI alignment, are very reliable. We performed an in silico benchmark of cell barcode assignment when both, cell barcode and UMI, are found in the Nanopore read. We observed an average specificity of $99.9 \%$ (ScNapBar) and $99.8 \%$ (Sicelore) over 100 averaged simulation runs (Fig. 2A). As expected, sensitivity heavily depends on Illumina sequencing saturation (Fig. 2A). As the UMI approach relies on consistent genomic mappings for the Illumina and Nanopore reads, other challenges include insufficient or inaccurate genome annotations causing wrong gene assignment; chimeric or super-long Nanopore reads assigned to multiple genes increase the risk of assigning a false UMI.

\section{The probabilistic approach of ScNapBar}

Complementary to the UMI approach, we implemented a Bayesian approach in ScNapBar, which covers the situation of low Illumina sequencing saturation. In our second approach, UMI alignments are no longer used. ScNapBar evaluates probability scores for each barcode alignment instead. Illumina sequencing saturation measures the uniqueness of the transcripts detected in the Illumina library. Given that we have performed Illumina and Nanopore sequencing in our approach, the lllumina sequencing saturation limits the overlap of cell barcodes and UMls with the low depth Nanopore libraries. To explore more realistic saturation scenarios, we estimated the Illumina sequencing saturation for our pilot data set with the Cell 

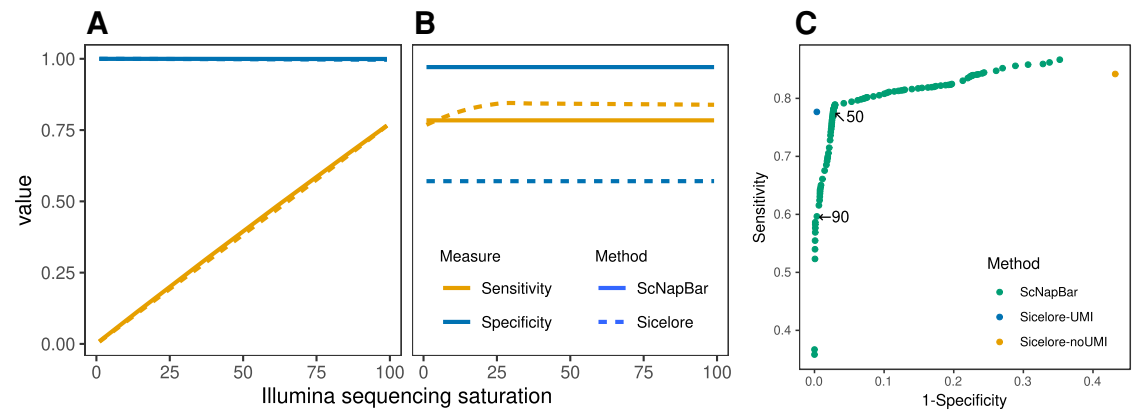

FIGURE 2. Sensitivity and specificity of ScNapBar and Sicelore on 100 Illumina libraries with different levels of saturation. (A) Barcode assignment with UMI matches. (B) Barcode assignment without UMI matches (ScNapBar score $>50$ ). (C) Benchmark of the specificity and sensitivity of the Illumina library with $100 \%$ saturation. We compared the barcode assignments with ScNapBar score $>1-99$, and the assignments from Sicelore with UMI support are roughly equivalent to the ScNapBar score $>90$.

Ranger software. Herein, sequencing saturation is calculated as

$$
\text { Saturation }=1-\left(n_{\text {deduped reads }} / n_{\text {reads }}\right) \text {, }
$$

where $n_{\text {deduped reads }}$ is the number of unique (valid cell-barcode, valid UMI, gene) combinations among confidently mapped reads and $n_{\text {reads }}$ is the total number of confidently mapped, valid cell-barcode, valid UMI reads. For example, we have observed a saturation of $11.3 \%$ for our pilot data set.

We have simulated one million Nanopore reads with an error model, which was estimated from our reference Nanopore libraries (see Materials and Methods) using the same gene-barcode-UMI composition as given by the Illumina library and a sequencing saturation of $100 \%$. We trained a Naïve Bayes classifier (see Materials and Methods) from barcode and adapter alignments of one Nanopore library and applied the model for computing the likelihood of the matched barcodes $P\left(r \mid b_{i}\right)$ on the other library. Then we used the frequencies of the given barcodes in the Illumina library as prior probabilities $P\left(b_{i}\right)$ and calculated the posterior probability $P\left(b_{i} \mid r\right)$ from the likelihood and prior probabilities. We scored each barcode alignment by multiplying the $P\left(b_{i} \mid r\right)$ by 100 and assigned the best matching barcode with the highest score $(>50)$ as the predicted barcode assignment. Using the probability scores as mentioned, ScNapBar correctly assigned $65.8 \%$ barcodes from one million simulated Nanopore reads, of which $26.5 \%$ contains at least one mismatch or indel (Supplemental Fig. S1).

We estimate a user data specific error model, simulate data from which users pick the Bayes score cutoff, which meets their requirements on sensitivity and specificity, respectively. We inspected the densities of the probability scores by examining the ground-truth barcodes and confirmed that the correct barcode assignments are enriched in high scoring barcodes (Supplemental Fig. S2b).
Our probabilistic model outperforms Sicelore for cases where UMI information is sparse and cannot be used to assign cell barcodes. In the absence of UMls, ScNapBar reaches 97.1\% specificity while Sicelore reaches only $57.1 \%$ (Fig. 2B).

We examined performance metrics of cell barcode assignment over a range of score cutoffs (from 1 to 99), and the specificity increases while the sensitivity decreases along with the increased thresholds (Supplemental Fig. S3). We pooled the simulated results from FC1 and FC2 together and use the Sicelore assignments as baselines. At some cutoff thresholds, ScNapBar has better F1 scores than Sicelore (e.g., cutoff $=50$ ), and ScNapBar score $>90$ is as accurate as Sicelore with UMI from the receiver-operating characteristic (ROC) graph (Fig. 2C).

\section{The runtime performance of ScNapBar}

ScNapBar is based on the Needleman-Wunsch algorithm (gap-end free, semiglobal sequence alignment) of FLEXBAR (Dodt et al. 2012; Roehr et al. 2017), and Sicelore is based on the "brute force approach," which hashes all possible sequence tag variants (including indels) up to a certain edit distance (2 or 3 ) of the given barcode sequences. The time complexity of ScNapBar and Sicelore can be represented as Equations $2 a$ and 2b, respectively:

$$
\begin{gathered}
T(n) \propto\left(I_{p o s}+I_{c b}\right) I_{c b} n_{c b}, \\
T(n) \propto \frac{\left(n_{p o s}+I_{c b}\right) !}{n_{e d} !} I_{p o s} n_{c b},
\end{gathered}
$$

where $n_{\text {pos }}$ is the number of nucleotides downstream from the adapter, and $I_{\text {pos }}=2 n_{\text {pos }}+1$ as Sicelore typically searches the same number of nucleotides upstream and downstream from the ending position of the adapter. $n_{c b}$ stands for the number of barcodes in the whitelist from Illumina sequencing. $n_{e d}$ is typically two or three as larger edit distances increase runtime drastically and are not necessarily due to the increasing error rate. $I_{c b}$ is the length of the barcode and is 16 in this study.

We compared the runtime between ScNapBar and Sicelore with regards to start positions of barcodes (number of nucleotides between adapter and barcode). We discovered that Sicelore may be orders of magnitude slower than ScNapBar given the same search space (2052 cellular barcodes, edit distance $=3$ ), but also its runtime increases exponentially as the barcode start position increases (Fig. $3 B$ ). Sincelore by default searches \pm 1 -nt from the end of the adapter, which may limit the nucleotides search space 
A

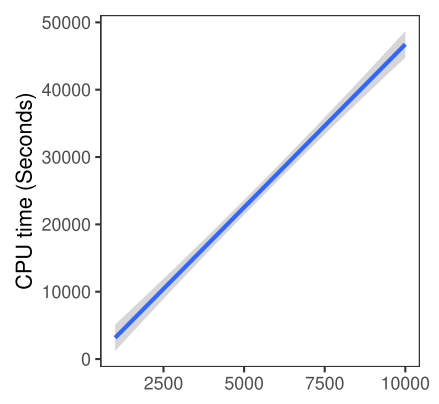

Number of barcodes in the white list
B

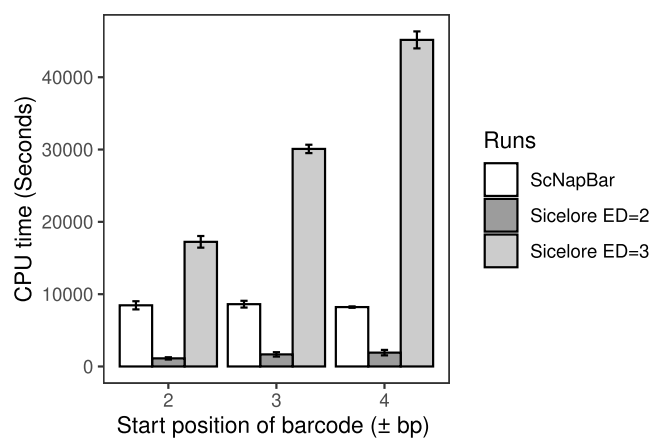

FIGURE 3. Sicelore and ScNapBar CPU time comparison. (A) ScNapBar CPU time depends on the number of whitelist barcodes (allowing an edit distance of $>2$ and and offset of up to $4 \mathrm{bp}$ between adapter and barcode). Gray area represents the standard deviation for 10 runs. (B) Comparison of ScNapBar and Sicelore CPU times. Benchmark was measured using one million barcode sequences and 2052 barcodes in the whitelist.

and could cause false assignments. Importantly, we did observe that $7.7 \%$ of all ground-truth barcodes have offsets $>1$-nt in the simulated read set, and $7.8 \%$ of all barcode assignments with $\mathrm{ScNapBar}$ score $\geq 50$ in real Nanopore reads. We assessed the impact of various factors (e.g., indels $\geq 3$ against $<3$ ) on cell barcode assignment accuracy using Fisher's test (see Supplemental Table S1). Our findings imply that larger offsets could effectively reduce the false-positive rate, which is feasible in less time with ScNapBar.

We also performed real runtime comparison on barcode assignment on the previously simulated one million Nanopore reads. In this test, we provided ScNapBar with ten barcode white lists, which contain from 1000 to 10,000 of the most abundant barcodes, and ScNapBar's runtime is only dependent on the number of barcodes to search given the other factors are fixed in this study (Fig. 3A). Then we tested Sicelore with searching parameters of barcode edit distance between two and three, barcode start position from $\pm 2 \mathrm{bp}$ to $\pm 4 \mathrm{bp}$, and UMl edit distance of 0 . ScNapBar requires only one-fifth CPU time than Sicelore when barcode start position $= \pm 4$ bp and barcode edit distance $=3$ are considered in both programs (Fig. 3B).

\section{The performance of ScNapBar on the real data}

\section{The performance of ScNapBar on an Illumina library with high sequencing saturations}

We tested our ScNapBar software with the UMI approach (option 1) on the data set from the Sicelore paper (NCBI GEO GSE130708). Herein, Illumina sequencing saturation reaches $\mathbf{9 0 . 5 \%}$. Similar to Sicelore, we extracted the UMI whitelists for each gene or genomic window (500 bp) from the Illumina library. The $500 \mathrm{bp}$ threshold is useful when a matched $\mathrm{UMI}$ is found in Illumina data for the same genomic region but not for the same gene. This situation may arise from incomplete gene annotations or mapping ambiguities. We observed that $99 \%$ of the Nanopore reads are within this $500 \mathrm{bp}$ window when comparing the mapping positions of the Illumina and Nanopore reads that have the same UMls in this data set. We set the minimum length of an UMI match to 7 in ScNapBar. In summary, Sicelore and ScNapBar assigned barcodes to $84.3 \%$ and $77.2 \%$ of the 9,743,819 Nanopore reads (Supplemental Fig. S4), respectively. $88.4 \%$ of the assigned barcodes are identical.

\section{The performance of ScNapBar on an Illumina library with low sequencing saturations}

We ran ScNapBar with the Bayesian approach (option 2) on our NMD data set, which only has an Illumina saturation of $11.3 \%$. ScNapBar assigns $35.0 \%$ and $36.3 \%$ of the Nanopore reads to cell barcodes with probability score $>50$, while Sicelore assigns $40.8 \%$ and $42.5 \%$ without using UMls ("Assigned to barcode" in Fig. 4) and only

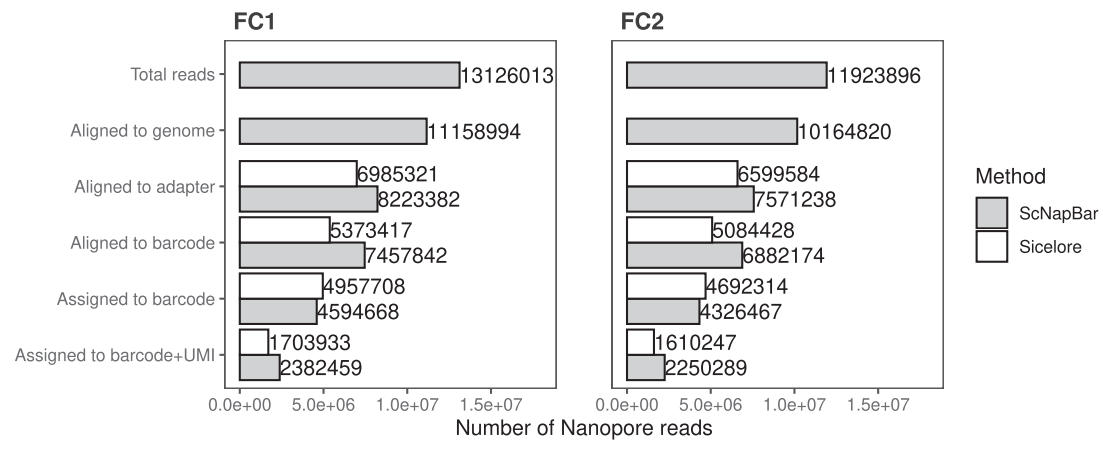

FIGURE 4. Number of the Nanopore reads identified by ScNapBar and Sicelore at each processing step. We inspected each processing step on real data (low Illumina saturation of 11.3\%). The first two steps are identical for both workflows. Total Reads: Number of input reads, aligned to genome: Number of reads aligned to genome. The next three steps are workflow-specific: Aligned to adapter: Number of reads with identified adapter sequence, aligned to barcode: Number of reads with aligned barcode sequence, Assigned to barcode: Number of predictions by each workflow. The last step is a validation of the previous assignment step after additional Illumina sequencing, which increases the Illumina saturation to $52 \%$, and using UMI matches, see main text. 
assigns $4.0 \%$ and $4.2 \%$ of the Nanopore reads using the UMI approach for FC1 and FC2, respectively. The correlations of the number of gene/ UMls for each cell between Illumina and Nanopore imply good matches from the ScNapBar assignments (Supplemental Fig. S5).

In order to validate the correctness of our cell barcode assignments, we have sequenced $\approx 485$ million additional Illumina reads from the same library. The saturation reaches $52 \%$ after combining both Illumina sequencing data (80.8 million reads from primary run and 485 million from secondary run). We searched UMIs in the Nanopore reads from the combined Illumina runs using both ScNapBar and Sicelore. We ran ScNapBar by allowing up to three edit distances between the UMls of the Nanopore and Illumina reads, and ran Sicelore with the default parameters (-maxUMIfalseMatchPercent 1 -maxBCfalseMatchPercent 5). CBCs and UMls must be matched for the same genomic region within a window of $500 \mathrm{nt}$ as the Illumina library. We observed $\approx 50 \%$ of the barcode assignments by ScNapBar have matched UMIs ("Assigned to barcode + UMI" in Fig. 4).

\section{Single-cell clustering and splicing in a pool of wild-type and NMD mutant cells}

Although alternative splicing increases the coding potential of the human genome, aberrant isoforms are frequently generated that contain premature termination codons (PTCs) (Lewis et al. 2003). Regular stop codons are normally located in the last exon of a transcript or at most $50 \mathrm{nt}$ upstream of the last exon-exon junction (Lindeboom et al. 2019). Alternative splicing can result in PTCs by exon inclusion/exclusion events or can convert normal stop codons into PTCs by splicing in the $3^{\prime}-$ UTR. Transcripts harboring PTCs are rapidly degraded by the nonsense-mediated mRNA decay (NMD) machinery, not only to remove faulty mRNAs, but also to fine-tune and regulate the transcriptome. $5 \%-40 \%$ of all expressed human genes are directly or indirectly altered in expression levels, splicing pattern, or isoform composition by the NMD pathway (Boehm et al. 2020). We have sequenced a pool of NMD active and inactive cells and expect to see an enrichment of transcripts with PTCs in GFP- cells.

We use the GFP label as an independent confirmation of cellular NMD status and pooled data from both experiments (FC1 and FC2). For the Nanopore data, Seurat identifies 13,807 expressed genes across 1850 cells. We extracted the GFP+ barcodes from the Illumina reads mapping and rendered the corresponding cells in different col- ors in the t-SNE plots (Fig. 5). The locations of the GFP+ cells appear in distinct subclusters in the Illumina and Nanopore t-SNE plots.

We characterized the structural changes of the assembled Nanopore transcripts based on our customized transcriptome annotations using NMD Classifier (Hsu et al. 2017). The pool of SMG7-KO/SMG6-KD (GFP-) cells harbors almost twice as many inclusion/exclusion events, which lead to the formation of a PTC (Supplemental Fig. S6a). We quantified the expression level of 14,185 known NMD transcripts annotated by Ensembl release 101. After removing the nonexpressed transcripts from both flow cell runs, the remaining $6423 \mathrm{NMD}$ transcripts have shown significantly higher NMD transcript expression in the SMG7KO/SMG6-KD (GFP-) cells than the WT (GFP+) cells (Supplemental Fig. S6b). We reason that the lowered NMD response is clearly visible by the enrichment of PTC-containing transcripts in the pool of SMG7-KO/ SMG6-KD (GFP-) cells. Consequently, the cell barcode assignments meet our "biological" expectations.

We investigated a well-established NMD target SRSF2 in detail (Sureau et al. 2001). The wild-type isoforms are present in both GFP+/- cells, while in the GFP- cells, the PTC-containing isoforms are more abundant in the GFP- cells (Supplemental Fig. S7a). The view on the SRSF2 genome locus confirmed the different splicing junctions between two cell types (Supplemental Fig. $\mathrm{S} 7 \mathrm{~b})$. The inclusion of exon 3 (middle) is clearly favored GFP- cells.

\section{DISCUSSION}

The current ecosystem of single-cell RNA-seq platforms is rapidly expanding, but robust solutions for single-cell and single-molecule full-length RNA sequencing are virtually absent. In our manuscript, we combined Oxford Nanopore single-molecule sequencing of $10 \times$ Genomics cDNA libraries and developed a novel software tool to arrive at single-cell, single-molecule, full cDNA length 
resolution. In contrast to Lebrigand et al. (2020), our Bayesian method for cell barcode assignment performs superiorly in situations of low sequencing saturation. Even in the light of expected improvements of ONT sequencing error rates, ScNapBar offers improved performance in the aforementioned use case (see Supplemental Fig. S8). In summary, we could track in a well-controlled setting, that is, by using GFP labeled cells and strong transcriptome perturbations, full-length transcript information at a single-cell level. We have identified differential RNA splicing linked to NMD pathway activity across our cell population. Our high-throughput full-length RNA sequencing solution is a necessary step forward toward studying the complex life of mRNA on a single-cell level. This opens up unprecedented opportunities in low saturation settings such as multiplexed CRISPR-based screens.

\section{MATERIALS AND METHODS}

\section{Single-cell samples preparation and experiment}

We performed an experiment using two different Flp-In-T-REx293 cell lines: the wild-type cell line with stably integrated FLAG-emGFP and a SMG7 knockout (KO) cell line (generated and established in Boehm et al. 2020). Wild-type cells (GFP+) were transfected with siRNA against Luciferase and the SMG7 KO cells (GFP-) were transfected with an siRNA against SMG6. Two days after siRNA transfection, we mixed both cell types at a 1:1 ratio with a target of 2000 cells in total. cDNA was prepared according to the $10 \times$ Genomics Chromium Single Cell 3' Reagent Kit User Guide (v3 Chemistry) from the pool of $\approx 2000$ cells with a yield of $1.68 \mu \mathrm{g}$ cDNA (42 ng/ $\mu \mathrm{L}$ concentration, $40 \mu \mathrm{L}$ volume) and a fragment size of $1.5 \mathrm{kbp}$. At this point, all cDNA fragments carry the $16 \mathrm{nt}$ cellular barcode and $12 \mathrm{nt} \mathrm{UMI}$ at the Poly (dT) end. $25 \%(10 \mu \mathrm{L})$ of this cDNA solution continued with the original $10 \times$ Genomics protocol to create an Illumina 3' mRNA library with P5 and P7 Illumina adapters, and paired-end reads of this library will present cellular barcodes and UMI with the first read and a $90 \mathrm{nt}$ second read containing $3^{\prime}$ mRNA sequence. Further on, we produced two ONT libraries with $200 \mathrm{ng}$ each of the same cDNA (0.2 pmoles) with the ONT Direct cDNA Sequencing Kit SOKDCS109 protocol according to the manufactures' standard procedures. We sequenced each library on one Gridlon flowcell (FLOMIN106D R9 Version/R9.4.1) creating reads of full cDNA length that contained the same compositions of $\approx 2000$ cellular barcodes as the Illumina data (based on the same cells) but a different composition of UMIs (different transcripts).

\section{Illumina reads processing and identification of cellular barcodes}

We used 10× Genomics Cell Ranger 3.1 (https://github.com/ 10XGenomics/cellranger) to map the Illumina reads onto the reference genome. In our NMD data set, the DNA sequences of luciferase were appended to the reference genome, and therefore the GFP+ cells can be called from Cell Ranger. Cell Ranger also corrects the sequencing errors in the barcode and unique molec- ular identifier (UMI) sequences. Cell Ranger estimates the number of cells using a Good-Turing frequency estimation model (https:// support.10xgenomics.com) and characterized the identified barcodes into the cell-associated and background-associated barcodes. We used the cell-associated barcode sequences as the cellular barcode whitelist in the following analyses. Our Cell Ranger analysis estimated 2052 sequenced cells (Supplemental Table S2). The read counts per cell of the estimated cell barcodes are shown in Supplemental Figure S9.

\section{Nanopore reads processing, mapping, and gene assignment}

We sequenced the two independently prepared Nanopore libraries from the same cDNA on two Nanopore R9.4 GridION flow cells (FC1 and FC2). The base-calling of Nanopore reads was done using Guppy v3.3.3, resulting in 13,126,013 and 11,923,896 reads, respectively. We aligned the Nanopore reads onto the corresponding reference genome using minimap2 v2.17 (Li 2018) in the spliced alignment mode (-ax splice). The two Nanopore runs yielded 11,158,994 and 10,164,820 mappable reads, respectively. We further assigned gene names to Nanopore reads using the "TagReadWithGeneExon" program from the Drop-seq tools (Macosko et al. 2015). We assembled all the Nanopore reads and extended transcriptome annotations using StringTie v2.1.1 (Pertea et al. 2015). The FPKM level of the assembled transcripts were quantified using Ballgown v2.14.1 (Frazee et al. 2015).

\section{Identification of the adapter, barcode, UMI, and poly(A)-tail sequences from Nanopore reads}

We removed the cDNA sequences from Nanopore reads and extracted up to $100 \mathrm{bp}$ from both ends. We developed a modified version of FLEXBAR (Dodt et al. 2012; Roehr et al. 2017) to align P1 primer adapter sequence with the following parameters ("-ao 10 -ae 0.3 -ag -2 -hr T -hi 10 -he 0.3 -be 0.2 -bg -2 -bo 5 -ul 26 -kb 3 $-f\left(100^{\prime \prime}\right)$. Then we aligned the Nanopore reads that have valid adapters to the cellular barcodes which have been previously identified by Cell Ranger. We scanned the poly(A) sequences using the homopolymer-trimming function of FLEXBAR downstream from the cell barcode. Once the poly(A) sequences were found, the UMI sequences between the poly $(A)$ and barcode were searched using MUMmer 4.0 (Marçais et al. 2018) (with parameters "-maxmatch $\left.-b-c-17-F^{\prime \prime}\right)$ and in-house scripts against the Illumina UMls of the same cell and the same gene or genomic regions ( \pm 500 bp from each end of the reads). In the end, ScNapBar output the alignment score of the adapter, the number of mismatches and indel from the barcode alignment, the length of poly(A) and UMI sequences, as well as the length of the gap between the barcode and adapter. We use these features to estimate the likelihood of the barcode assignment in the steps shown in Figure 1.

\section{Simulation and engineering of discriminative features from the barcode and adapter alignments}

We characterized the correct and false barcode assignment by simulating Nanopore reads. We created some artificial template 
sequences which contain only the P1 primer, cellular barcode, and UMI sequences at the same frequencies as the Illumina library, followed by 20 bp oligo(dT) and 32 bp cDNA sequences. In the next step, we first used NanoSim (Yang et al. 2017) to estimate the error profile of our Nanopore library, then we generated one million Nanopore reads from the artificial template using the NanoSim simulator with the previously estimated error profile. We aligned the simulated Nanopore reads to the adapter and barcode sequences using ScNapBar. We compared the sequences in the simulated Nanopore reads and the sequences from the artificial template and labeled the assigned barcode as correct or false accordingly. By comparing sequence and alignment features [adaptor score, poly $(T)$ length, barcode indel, barcode mismatch, barcode start] of correct and false assignments, we found that the two categories (false, true) could be discriminated by these features (Supplemental Fig. S10c). We then assessed the importance of each feature toward the correctness of the assignment (Supplemental Fig. S10a). As these features are uncorrelated (Supplemental Fig. S10b), we train a Naïve Bayes model from these features to predict the likelihood of the correctness of a barcode assignment.

\section{Calculate cell barcode posterior probability using prior probabilities from the Illumina data set}

We denote $b_{1}, b_{2}, \ldots, b_{n}$ as barcodes that match to read $r$ and define $P\left(b_{1} \mid r\right)$ as the probability that barcode $b 1$ was sequenced given $r$ is observed. Following Bayes' theorem, $P\left(b_{1} \mid r\right)$ could be computed as in Equation 3a, and further computed as in Equation $3 \mathrm{~b}$ according to the total probability theorem:

$$
\begin{gathered}
P\left(b_{1} \mid r\right)=\frac{P\left(r \mid b_{1}\right) P\left(b_{1}\right)}{P(r)}, \\
=\frac{P\left(r \mid b_{1}\right) P\left(b_{1}\right)}{P\left(r \mid b_{1}\right) P\left(b_{1}\right)+\ldots+P\left(r \mid b_{n}\right) P\left(b_{n}\right)},
\end{gathered}
$$

where $P\left(r \mid b_{1}\right)$ and $P\left(r \mid b_{n}\right)$ are computed by the Naïve Bayes predictor, and priors $P\left(b_{1}\right)$ and $P\left(b_{n}\right)$ can be estimated from the observed barcode counts in Illumina sequencing. For practical reasons, as the probabilities for the unaligned barcodes that contain a lot of mismatches are pretty low, we add a pseudocount of one to the denominator to represent them. Because we have sequenced the same library twice using the Nanopore and Illumina sequencer, we assume prior probabilities $P(b)$ are the same for the Nanopore and the Illumina platform (Supplemental Fig. S2a).

\section{Quality assessment and clustering of the single-cell libraries}

A metagene body coverage analysis confirmed the near fulllength character of the Nanopore approach (Supplemental Fig. S11a). After assigning gene names and cell barcodes to the Nanopore reads, we processed the gene-barcode expression matrix using Seurat v3.1.1 (Butler et al. 2018) by keeping all genes that are expressed in at least three cells, and cells with more than 200 genes expressed. We then scaled the expression matrix by a factor of 10,000 and log-normalized and performed the tSNE analysis.

\section{DATA DEPOSITION}

All sequencing data were deposited in NBCl's BioProject database (BioProject ID PRJNA722142). ScNapBar workflow (code and tutorial) is available at https://github.com/dieterich-lab/ single-cell-nanopore.

\section{SUPPLEMENTAL MATERIAL}

Supplemental material is available for this article.

\section{ACKNOWLEDGMENTS}

The authors would like to thank Etienne Boileau, Thiago Britto Borges, Tobias Jakobi for proofreading and comments. The authors are grateful to Marek Franitza for running the experiments on the 10x platform and to Christian Becker for running ONT sequencing. This work was supported by Informatics for Life funded by the Klaus Tschira Foundation.

Author contributions: Q.W. implemented the ScNapBar workflow, performed data analyses, and wrote the manuscript. S.B. modified the FLEXBAR implementation. V.B. performed cell culture experiments and helped to draft the manuscript. N.G. helped to draft the manuscript and acquired funding. J.A. performed all sequencing experiments and helped to draft the manuscript. C.D. supervised the project, performed data analyses, acquired funding, and wrote the manuscript.

Received October 16, 2020; accepted April 20, 2021.

\section{REFERENCES}

Boehm V, Kueckelmann S, Gerbracht JV, Britto-Borges T, Altmüller J, Dieterich C, Gehring NH. 2020. Nonsense-mediated mRNA decay relies on "two-factor authentication" by SMG5-SMG7. bioRxiv doi: 10.1101/2020.07.07.191437

Butler A, Hoffman P, Smibert P, Papalexi E, Satija R. 2018. Integrating single-cell transcriptomic data across different conditions, technologies, and species. Nat Biotechnol 36: 411-420. doi:10.1038/nbt .4096

Byrne A, Beaudin AE, Olsen HE, Jain M, Cole C, Palmer T, DuBois RM, Forsberg EC, Akeson M, Vollmers C. 2017. Nanopore long-read RNA-seq reveals widespread transcriptional variation among the surface receptors of individual B cells. Nat Commun 8: 16027. doi:10.1038/ncomms 16027

Cole C, Byrne A, Adams M, Volden R, Vollmers C. 2020. Complete characterization of the human immune cell transcriptome using accurate full-length cDNA sequencing. Genome Res 30: 589-601. doi:10.1101/gr.257188.119

Dodt M, Roehr JT, Ahmed R, Dieterich C. 2012. FLEXBAR-flexible barcode and adapter processing for next-generation sequencing platforms. Biology (Basel) 1: 895-905. doi:10.3390/ biology 1030895

Döring A, Weese D, Rausch T, Reinert K. 2008. SeqAn: an efficient, generic $\mathrm{C}++$ library for sequence analysis. BMC Bioinformatics 9: 11. doi:10.1186/1471-2105-9-11

Frazee AC, Pertea G, Jaffe AE, Langmead B, Salzberg SL, Leek JT. 2015. Ballgown bridges the gap between transcriptome assembly and expression analysis. Nat Biotechnol 33: 243-246. doi:10 .1038/nbt.3172 
Hsu MK, Lin HY, Chen FC. 2017. NMD Classifier: a reliable and systematic classification tool for nonsense-mediated decay events. PLOS ONE 12: e0174798. doi:10.1371/journal.pone.0174798

Kent WJ. 2002. BLAT-The BLAST-like alignment tool. Genome Res 12: 656-664. doi:10.1101/gr.229202

Krehenwinkel H, Pomerantz A, Henderson JB, Kennedy SR, Lim JY, Swamy V, Shoobridge JD, Graham N, Patel NH, Gillespie RG, et al. 2019. Nanopore sequencing of long ribosomal DNA amplicons enables portable and simple biodiversity assessments with high phylogenetic resolution across broad taxonomic scale. Gigascience 8: giz006. doi:10.1093/gigascience/giz006

Lebrigand K, Magnone V, Barbry P, Waldmann R. 2020. High throughput error corrected Nanopore single cell transcriptome sequencing. Nat commun 11: 4025. doi:10.1038/s41467-020-17800-6

Lewis BP, Green RE, Brenner SE. 2003. Evidence for the widespread coupling of alternative splicing and nonsense-mediated mRNA decay in humans. Proc Natl Acad Sci 100: 189-192. doi:10 .1073/pnas.0136770100

Li H. 2018. Minimap2: pairwise alignment for nucleotide sequences. Bioinformatics 34: 3094-3100. doi:10.1093/bioinformatics/ bty191

Lindeboom RGH, Vermeulen M, Lehner B, Supek F. 2019. The impact of nonsense-mediated mRNA decay on genetic disease, gene editing and cancer immunotherapy. Nat Genet 51: 1645-1651. doi:10.1038/s41588-019-0517-5

Macosko EZ, Basu A, Satija R, Nemesh J, Shekhar K, Goldman M, Tirosh I, Bialas AR, Kamitaki N, Martersteck EM, et al. 2015. Highly parallel genome-wide expression profiling of individual cells using nanoliter droplets. Cell 161: 1202-1214. doi:10 .1016/j.cell.2015.05.002

Marçais G, Delcher AL, Phillippy AM, Coston R, Salzberg SL, Zimin A. 2018. MUMmer4: a fast and versatile genome alignment system.
PLoS Comput Biol 14: e1005944. doi:10.1371/journal.pcbi .1005944

Pertea M, Pertea GM, Antonescu CM, Chang TC, Mendell JT, Salzberg SL. 2015. StringTie enables improved reconstruction of a transcriptome from RNA-seq reads. Nat Biotechnol 33: 290295. doi:10.1038/nbt.3122

Roehr JT, Dieterich C, Reinert K. 2017. Flexbar 3.0: SIMD and multicore parallelization. Bioinformatics 33: 2941-2942. doi:10.1093/ bioinformatics/btx330

Smith MA, Ersavas T, Ferguson JM, Liu H, Lucas MC, Begik O, Bojarski L, Barton K, Novoa EM. 2020. Molecular barcoding of native RNAs using nanopore sequencing and deep learning. Genome Res 30: 1345-1353. doi:10.1101/gr.260836.120

Sureau A, Gattoni R, Dooghe Y, Stévenin J, Soret J. 2001. SC35 autoregulates its expression by promoting splicing events that destabilize its mRNAs. EMBO J 20: 1785-1796. doi:10.1093/ emboj/20.7.1785

Volden R, Vollmers C. 2020. Highly multiplexed single-cell full-length cDNA sequencing of human immune cells with $10 X$ Genomics and R2C2. bioRxiv doi: 10.1101/2020.01.10.902361

Volden R, Palmer T, Byrne A, Cole C, Schmitz RJ, Green RE, Vollmers C. 2018. Improving nanopore read accuracy with the R2C2 method enables the sequencing of highly multiplexed fulllength single-cell cDNA. Proc Natl Acad Sci 115: 9726-9731. doi:10.1073/pnas.1806447115

Wick RR, Judd LM, Holt KE. 2018. Deepbinner: demultiplexing barcoded Oxford Nanopore reads with deep convolutional neural networks. PLoS Comput Biol 14: e1006583. doi:10.1371/journal pcbi.1006583

Yang C, Chu J, Warren RL, Birol I. 2017. NanoSim: nanopore sequence read simulator based on statistical characterization. Gigascience 6: 1-6. doi:10.1093/gigascience/gix089 

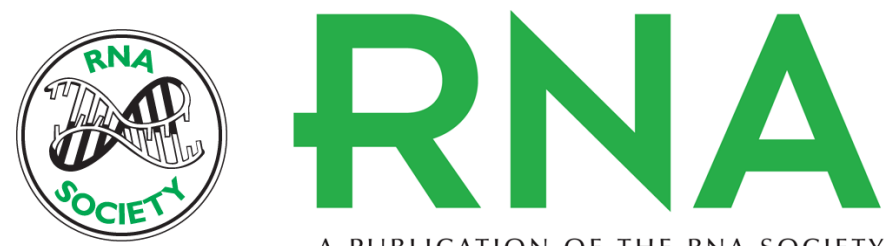

A PUBLICATION OF THE RNA SOCIETY

\section{Single-cell transcriptome sequencing on the Nanopore platform with ScNapBar}

Qi Wang, Sven Bönigk, Volker Böhm, et al.

RNA 2021 27: 763-770 originally published online April 27, 2021

Access the most recent version at doi:10.1261/rna.078154.120

\section{Supplemental http://rnajournal.cshlp.org/content/suppl/2021/04/27/rna.078154.120.DC1 \\ Material}

References This article cites 24 articles, 8 of which can be accessed free at: http://rnajournal.cshlp.org/content/27/7/763.full.html\#ref-list-1

Open Access Freely available online through the RNA Open Access option.

Creative This article, published in $R N A$, is available under a Creative Commons License Commons (Attribution-NonCommercial 4.0 International), as described at

License http://creativecommons.org/licenses/by-nc/4.0/.

Email Alerting Receive free email alerts when new articles cite this article - sign up in the box at the Service top right corner of the article or click here. 